\title{
Does weak discernibility determine metaphysics?
}

\author{
Jonas R. Becker ArenharT
}

Received: 16/02/2016

Final Version: 04/07/2016

BIBLID 0495-4548(2017)32:1p.109-125

DOI: $10.1387 /$ theoria. 15870

ABSTRACT: Two entities are weakly discernible when there is an irreflexive and symmetric relation holding between them. That weak discernibility holds in quantum mechanics is fairly uncontroversial nowadays. The ontological consequences of weak discernibility, however, are far from clear. Recently, part of the literature seems to imply that weak discernibility points to a definite metaphysics to quantum mechanics. In this paper we shall discuss the metaphysical contribution of weak discernibility to the metaphysics of quantum mechanics and argue that, contrary to part of current literature, it does not provide for a fully naturalistic determination of the theory's metaphysics. Underdetermination still obtains.

Keywords: weak discernibility; ontic structural realism; naturalistic metaphysics; metaphysical underdetermination.

RESUMEN: Dos entidades son débilmente discernibles cuando entre ellas se mantiene una relación irreflexiva y simétrica. En la actualidad no se discute que la discernibilidad débil se da en la mecánica cuántica. Las consecuencias ontológicas de la discernibilidad débil, sin embargo, no están muy claras. Parte de la literatura al respecto parece implicar que la discernibilidad débil apunta, en la mecánica cuántica, a una metafísica determinada. En este artículo analizaremos la contribución metafísica de la discernibilidad débil a la mecánica cuántica y argumentaremos que, al contrario de lo que se sostiene en parte de la literatura actual, la discernibilidad débil no proporciona una determinación completamente naturalista de la metafísica. La infradeterminación de la metafísica acecha aún en el camino del naturalista.

Palabras clave: discernibilidad débil, realismo estructural óntico, metafísica naturalista, infradeterminación metafísica.

\section{Introduction}

Traditionally, quantum entities were thought to be indiscernible: two quantum entities share every property and every relation. That leads directly to a violation of the Principle of the Identity of Indiscernibles (PII) in quantum mechanics. The violation of PII, on its turn, leads immediately to two distinct possibilities on what concerns the metaphysical status of its entities: they are either non-individuals (i.e., entities without identity conditions) or else they are indiscernible individuals; that is, entities individuated by some Transcendental Principle of Individuality (TI), like bare particulars, primitive thisnesses, haecceities and the like (see French and Krause 2006, chap. 4). That much seems to be uncontroversial in the traditional metaphysical analyses of quantum entities.

Still according to a more recent tradition, the fact that we have at least two options leads directly to a form of metaphysical underdetermination: quantum mechanics provides no hint as to which of the previous metaphysical packages should be preferred (French and Krause 2006, chap. 4, French 2011). That fact, obviously, puts some pressure on metaphysical naturalists, whose goal is to somehow infer our metaphysics from our best scientific theories. Here, metaphysical naturalism is to be understood roughly as Maudlin $(2007,104)$ puts it: 
Evidence for what exists, at least in the physical world, is provided solely by empirical research. Hence the proper object of most metaphysics is the careful analysis of our best scientific theories (and especially of fundamental physical theories) with the goal of determining what they imply about the constitution of the physical world.

The upshot of metaphysical underdetermination is that physics, or at least quantum mechanics, cannot do precisely that task on what concerns the nature of quantum particles (and in this paper we shall confine ourselves to that particular discussion).

Both of those theses (i.e. quantum indiscernibility and metaphysical underdetermination) have been recently under attack from the same front: weak discernibility in quantum mechanics. Beginning with Saunders (2003 and 2006), and then later with Muller and Saunders (2008), and Muller and Seevinck (2009) (with some corrections by Hugget and Norton 2014), the idea that quantum particles are indiscernible was deemed incorrect on the grounds that quantum theory furnishes relations that are able to weakly discern those particles. Recall, to say that $a$ and $b$ are weakly discernible means that there is an irreflexive and symmetric relation holding between $a$ and $b$. So, according to those authors, instead of being violated by quantum mechanics, the PII is rather vindicated in the theory (to be sure, there are further strategies to save PII for particles in non-entangled states that do not require weak discernibility; for discussions and references, see for instance Friebe 2014).

The second traditional thesis - metaphysical underdetermination - was also attacked with the apparatus of weak discernibility. Indirectly, the claim may be found in many of the writings of the defenders of weak discernibility, but it was Muller (2011 and 2014) who has put the issue most forcefully and explicitly. According to Muller, rather than providing for metaphysical underdetermination, quantum mechanics, due to weak discernibility, endorses a form of determination of metaphysics. The theory has metaphysical content, derived from the fact that weak discernibility holds. Instead of not being able to decide between non-individuals and individuals obeying Transcendental Individuality, the theory supports a metaphysics of weakly discernible entities. So, metaphysical naturalism is correct after all.

In this paper we shall discuss the idea that weak discernibility may provide determinate metaphysical content to quantum mechanics, at least on what concerns the nature of its fundamental entities. Our main claim is that metaphysical naturalism is not that easy to rescue in this specific case, and that there are many distinct and incompatible ways to put metaphysical flesh on the bare relational bones of weak discernibility. Indeed, it is possible to understand weak discernibility as substantiating both non-structural ontologies as well as structural ones. On the structuralist side, ontologies vary according to how one understands the dependence of objects on relations; there are currently at least three options, all compatible with weak discernibility. Weak discernibility and quantum mechanics are not enough to provide reasons for choosing only one of the approaches. On the non-structuralist side, there are at least two options, one providing for a Quinean ontology of individuals and the other for a more robust approach according to which individuals are "bundles" constituted by the properties and relations they bear. In the end, what emerges is that we must have the resources of typical metaphysical discussions if we are supposed to break the underdetermination.

The plan of the paper is as follows. In section 2 we rehearse important terminology and discuss how weak discernibility is expected to determine a metaphysics for quantum me- 
chanics. In section 3 we discuss how weak discernibility may generate more metaphysical underdetermination. In particular, we focus on the work of some proponents of weak discernibility. In section 4, we comment on the impact of our discussion for the prospects of a naturalized metaphysics. It is difficult to see how underdetermination could be broken with strictly physical means. We conclude in section 5 .

\section{Weak discernibility and metaphysical determination}

\subsection{Terminology}

As we have roughly set the issue in the previous section, metaphysical underdetermination arises because quantum mechanics cannot judge between two classes of metaphysical packages on what concerns the nature of its objects: a package according to which those objects are non-individuals (Krause and Arenhart 2015) and a package according to which they are individuals bearing some form of Transcendental Individuality (French and Krause 2006, chap. 4). Both approaches respect the traditional claim that quantum entities are indiscernible.

According to the proponents of weak discernibility, this discussion leaves one important (albeit crucial) issue behind: discernibility may come in distinct degrees. So far, all that was shown by followers of the traditional approach is that if particles $a$ and $b$ are of the same kind, then given any relation $\mathrm{R}$, if $\mathrm{R} a b$, then also $\mathrm{R} b a$; that is, a relation cannot distinguish its relata by the order in which they are related. However, - and that is the crucial point - that does not exhaust all the possibilities. As Saunders (2003) has first pointed out (followed by developments in Saunders 2006, Muller and Saunders 2008, and Muller and Seevinck 2009, and corrections by Huggett and Norton 2014), one can distinguish items $a$ and $b$ by providing a relation $\mathrm{R}$ which is symmetric and irreflexive: irreflexivity grants us that when Rab holds, $a$ cannot be the same as $b$, and symmetry grants us that the order of the relata is irrelevant for doing the discerning, given that for a symmetric $\mathrm{R}$ both Rab and $\mathrm{R} b a$ hold. Saunders, Muller, and Seevinck, in their works, have argued at length that quantum particles are physically discernible by such relations; indeed, those relations are furnished by the formalism of quantum mechanics itself.

To advance the relevant terminology in a nutshell: consider two items $a$ and $b$. We call them absolutely discernible when there is a property $\mathrm{P}$ that only one of them has, but the other does not have. Quantum particles of the same kind are not absolutely discernible. We say $a$ and $b$ are relatively discernible when there is a relation $\mathrm{R}$ such that one of $\mathrm{R} a b$ or $\mathrm{R} b a$ holds, but not both (that is, in this case it is the order of the relata that matters). Quantum particles of the same kind are not relatively discernible either. Finally, $a$ and $b$ are weakly discernible when there is a relation $\mathrm{R}$ that is irreflexive and symmetric holding between $a$ and $b$. Quantum particles of the same kind, it is said, are weakly discernible (for a discussion on the degrees of discernibility, see Muller and Saunders 2008, Muller and Seevinck 2009). For example, the relation "to have spin opposite to" weakly discerns fermions, since no fermion has spin opposite to itself in a given direction. To complete the list, $a$ and $b$ are indiscernible when they are not discernible in any one of the three previously mentioned ways. On this approach, only indiscernibles violate PII; but again, quantum particles are not indiscernibles: they are weakly discernible or (synonymously) relationals (the terminology is from Muller and Saunders 2008, Muller 2011). 
That much is thought to be enough to completely rule out the traditional view on quantum indiscernibility. The claim that weak discernibility is a kind of discernibility is itself contentious, but we shall not discuss that issue here (see for instance Ladyman and Bigaj 2010). Our focus will be on the stronger claim we shall present in the next section, according to which weak discernibility determines the metaphysics of quantum entities, i.e. it determines the metaphysical nature of those entities.

Before we proceed, however, as a parenthetical remark, one may be worried about the use of labels in the definitions of the degrees of discernibility. What is the role of labels? Certainly labels may be understood in a plurality of ways, and the role of names and labels in quantum mechanics is a very problematic issue (for further discussion see French and Krause 2006, chap. 5). Here, for the sake of argument, we shall proceed as Muller and Saunders (2008) do, assuming that labels act as some kind of content-free numerals, playing no distinctive metaphysical role. With that we may concentrate on the issue of underdetermination, leaving the issue of the use of labels in quantum metaphysics for another time (but see Ladyman, Linnebo and Pettigrew 2012 for a discussion of the role of labels in discernibility).

\subsection{Metaphysical determination}

Saunders (2003 and 2006) was the first to apply weak discernibility in quantum mechanics. The original project was designed to show not only that the orthodoxy is mistaken on what concerns the indiscernibility of quantum particles; in fact, Saunders also wanted to grant that, by having identity conditions, fermions could be considered as objects according to a thin notion of object. Roughly speaking, thin objects should be understood as the values of variables of quantification, as the referents of singular terms, the bearers of predicates. This notion of objecthood goes back at least to Quine, according to whom no entity should be admitted that does not have identity conditions (the famous "no entities without identity" slogan). By providing for weak discernibility in quantum mechanics, Saunders thinks fermions are now thought of as admissible objects according to this tradition.

In a nutshell, the project is developed by Saunders as follows. We begin with quantum mechanics as an interpreted physical theory; by abstraction we somehow manage to obtain the relations of the theory. These relations will comprise the finite non-logical vocabulary of a first-order theory without identity, and also with no individual constants and no function symbols. Now, inside this regimented language, due to its having only a finite non-logical vocabulary, we may define identity using a Quine-style definition as permutation invariance through all the predicates of the language (see Saunders 2003 and 2006 for details). As it is known, this definition of identity delivers the two usual axioms for identity in firstorder languages, i.e. reflexivity and the substitution law.

Saunders uses this language to show that a version of the PII holds in quantum mechanics for fermions: the numerical diversity of fermions $x$ and $y$ is backed by the existence of a relation $\mathrm{R}$ weakly discerning them. This version of PII is used as a methodological tool in order to account for what counts as objects and what does not. That is, for anything to be admitted in the domain of quantification (and, therefore, to be an object of the theory) it must first have identity conditions, in the sense that it must obey the PII. Since fermions do that, they are objects, they belong to the domain of quantification. Whatever fails the PII —as Saunders thought bosons did - do not count as a legitimate object in the do- 
main of quantification. So, instead of furnishing a counterexample to PII, bosons should be understood rather as excitation number of a certain mode of a quantum field, which on its turn is the real entity.

In this sense, weak discernibility is used, as Saunders $(2003,292)$ puts it, to tailor the metaphysics to suit the physics. We read off the metaphysics that is legitimately grounded by the physics, meaning precisely that the objects of the theory are those satisfying weak discernibility or, when available, stronger forms of discernibility. This expresses nicely the idea that weak discernibility is thought of as providing for ontological content being somehow derived or extracted from physics. Furthermore, Saunders $(2006,60)$ adds that fermions are individuals, given that it is not clear to him what else should an object do in order to be an individual (this conflation of objecthood with individuality will be recalled in what follows). That is, being an object already grants that the item in question is an entity and an individual; perhaps those expressions are synonymous (at least, Saunders seems to advance this idea).

The idea of tailoring the metaphysics to suit the physics is a first clear attempt to "read off" a metaphysics for quantum mechanics from physical relations obtaining in quantum theory. Given our physics and the weakly discerning relations that follow from it, there we have the constraints on what objects there are and, possibly, on their metaphysical profile (i.e. they are individuals). Weak discernibility allows us to extract some ontological juice from quantum theory. Muller (2011 and 2014) takes this idea even further and sees here that quantum mechanics is in direct conflict with metaphysical underdetermination, as presented before. Given that weak discernibility is derived from quantum mechanics, is that the end of metaphysical underdetermination?

According to Muller (2011 and 2014), the answer to that question is obviously "yes". Quantum mechanics vindicates a form of Determination Thesis (2011, 230):

Determination thesis: scientific theories are incompatible with some specific metaphysical views; in other words, they do have some definite ontological content.

That thesis is arrived at by following a simple reasoning: it is a quantum mechanical fact that quantum entities are relationals, i.e. weakly discernible entities. Given that, the first horn of the traditional metaphysical underdetermination, according to which quantum entities are indiscernible non-individuals, fails. The second horn, according to which quantum entities are indiscernible individuals, is motivated only as far as the first horn is a live option: it is only in order to avoid a metaphysics of indiscernible non-individuals that one is entitled to posit bare particulars, primitive thisnesses, and the like (the TI principles of individuation, recall). Given that the first horn is flawed (due to weak discernibility), there is no motivation for the second, and it may as well be abandoned.

In that scenario, what is left for us, metaphysically speaking? Well, there is only one option left: quantum objects are relationals. What else can be said about them, about their metaphysical status? By definition, relationals are entities whose discernibility depends solely on relations. Quantum mechanics tells us they are not absolutely discernible (recall the terminology in section 2.1). Having rejected TI forms of individuality, what we are left with is the fact that the identity of relationals somehow depends on the discerning relations, which on its turn amounts precisely to adopt a form of ontic structural realism (OSR), i.e. the view according to which we should give metaphysical primacy to relations over objects. In other words: weak discernibility, by being derivable from quantum 
mechanics, establishes that the metaphysical content of the theory is bound to be a form of OSR (for more on the characterization of OSR, see Ainsworth 2010; recall also that "metaphysical content" here concerns the nature of the entities dealt with by the theory). Consequently, metaphysical naturalism is also vindicated.

Now, how should we take that line of thought? We think that there are still some problems if naturalism is to be vindicated. For this moment, we would like to begin by pointing to an ambiguity in the statement of the Determination Thesis itself. To say that "scientific theories are incompatible with some specific metaphysical views" is not the same as saying that "they do have some definite ontological content". Indeed, those are two different statements which only in some circumstances may (accidentally) describe the same situation. Let us separate the two claims involved in the Determination Thesis:

- Negative determination thesis (NDT): scientific theories are incompatible with some specific metaphysical views.

- Positive determination thesis (PDT): scientific theories have some definite ontological content.

The NDT part is fairly uncontroversial and may be tied to a mitigated form of metaphysical naturalism, if naturalism that be (it was also advanced in Arenhart 2012 and in Chakravartty 2013, for instance). To keep with the case of quantum mechanics, NDT concerns the idea that quantum theory, for instance, is not compatible with some specific views on individuality. To consider the typical example (see also Arenhart 2012 for further discussion), it is not compatible with the view that particles are individuated by intrinsic properties (this amounts, roughly, in our terminology of section 2.1, to saying that they are not absolutely discernible). However, this is still very far from saying what quantum entities effectively are, metaphysically speaking. More is needed to do that and grant that a robust version of metaphysical naturalism holds. In fact, if the NDT was expected to determine what quantum entities are, we would need to grant that the theory is incompatible with all but one metaphysical view, and this is far from being the case (as we shall argue in the next section).

The goal of PDT seems precisely to ensure that there is one metaphysical view that may be singled out from a theory. A reading of PDT could be advanced so as to grant that it does not fix one metaphysical view, but allows room for multiple metaphysical views. That is, the "some", in "some definite ontological content" should be taken as allowing some room for distinct metaphysical views; what is relevant, according to this reading, is that the theory is not compatible with every metaphysical view. That reading would not, however, be enough to prevent metaphysical underdetermination and to ensure metaphysical determination. So, we shall take PDT as stating that it not only grants that some forms of metaphysical content are eliminated, but also that it attempts to establish that all but one form is the correct one. As we have seen, Muller argues that such content is precisely OSR.

So, we have illustrated how weak discernibility is used to determine a metaphysics for quantum mechanics. Talk about tailoring the metaphysics to suit the physics and about a determination thesis seem to point to the fact that weak discernibility grants specific metaphysical content to quantum mechanics. In the next section we shall survey the possible articulations of OSR compatible with weak discernibility and argue that it is hard to take weak discernibility as determining the metaphysics, even if we allow that the de- 
termination is taken in very broad lines, as a determination of a kind of metaphysics instead of the determination of one specific metaphysical view. More than that, as we shall see, there are even non-structural options that weak discernibility does not seem able to rule out as possible metaphysical candidates. So, in the end, metaphysical underdetermination strikes back.

\section{Weak discernibility and its metaphysics}

Recall that in the previous section we have presented not only Saunders' attempts at extracting some metaphysics from weak discernibility in quantum mechanics, but also Muller's attempt at debunking the underdetermination of metaphysics by the physics thesis. On the one hand, Saunders' view comprises relations and individuals. Muller's strategy involves arguing that quantum mechanics and weak discernibility point to OSR. Are those two metaphysical packages even compatible? Don't we already have a kind of underdetermination?

In this section we shall argue that even if those packages are compatible, the fact that the very question of their compatibility arises already raises many intricate issues that cannot be answered by the meager metaphysical resources provided by weak discernibility. More must be said about the relations between objects and relations in the context of OSR in order for us to have a precisely determined metaphysics of quantum entities. Of course, that extra dose of metaphysics does not come from quantum mechanics, and it is here that underdetermination finds its way back, just as does a considerable amount of non-naturalistic metaphysics.

\subsection{Non-structuralist interpretations}

The claim that weak discernibility may be employed to break metaphysical underdetermination was already considered before by French (2011). However, as French argues in that paper, that strategy needs not necessarily be seen as associated with OSR. The first option for the defender of weak discernibility would be to take Saunders' suggestion that fermions are individuals and attempt to ground such individuality in the success of the rehabilitated PII. Of course, one could maintain that it is precisely to this grounding that Saunders points to, although he does not go into the details. How does that view differ from Muller's suggestion that weak discernibility vindicates OSR?

As French puts it (French 2011, 216; see also French 2010, 106), this first reading of the situation takes the weak discernibility as contributing to a traditional understanding of individuality according to a form of bundle theory. According to this approach, an individual is a bundle of properties and relations tied together by a relation of co-presence (obviously, as usual the details will depend on how the "bundle" metaphor is precisely understood). Bundle theories are understood here as the theories of individuation that are traditionally opposed to TI forms of individuality: while the former argues that the individuality of a particular may be understood exclusively in terms of its qualities, the latter requires some extra non-qualitative ingredient to individuate (the TI ingredient, of course; the dialectics of the debate is briefly illustrated in French and Krause 2006, chap. 1, where further references may be found). So, seen in this context, this version of the bundle theory contributes to ground individuality in a "thick" sense; there are objects and their identity is determined by the relations and properties comprising their bundle. 
To put the matter succinctly, in this interpretation what is at issue is that the friend of weak discernibility is seen as attempting to ground individuality according to a traditional bundle theory, now allowing relations in the scope of quantifiers of the PII. Recall that the PII states that no two entities share every quality. The typical problem is to determine what counts as a legitimate quality that could be allowed in the scope of the quantifier in "every quality": only properties? Relations too? The issue of the scope of the quantifier is important because those qualities allowed to be quantified over are the ones responsible for the individuals' identity. So, according to the version of the bundle theory allowed by the weak version of the PII, when we allow weakly discerning relations in the scope of the quantifiers, fermions are individuals. So, this position goes back to the traditional notion of bundle individuality that was thought to be ruled out by quantum mechanics. The problem for those adhering to this metaphysical package, of course, is to justify that relations do indeed help individuate objects (see the discussions in French and Krause 2006). However difficult that may be, weak discernibility may be seen as contributing to that particular view.

Obviously, one could advance one step further in metaphysical abstraction and attempt to classify the relations and properties into universals, tropes, or both. That would lead us into the discussion of the appropriate categories to deal with those entities, but we shall not attempt that discussion here.

For the sake of reference, let us call that view M1, the first metaphysical package related with weak discernibility. This is not to be understood in the context of OSR, but rather in the context of the traditional dispute between so-called "constituent ontologies": bundle theories on the one hand, TI principles of individuation on the other (as we mentioned, they typically appear now in the literature as "thick individuals", theories of individuality outside the context of OSR; see also French 2010 and 2011 for thick and thin individuals). Individuals are somehow constituted by their properties and the relations they bear (given that we are assuming that relations may contribute to individuality). PII then grants that no two individuals have the same properties and/or bear the same relations. In fact, if $x$ and $y$ are weakly discernible, there is a relation $\mathrm{R}$ such that $x$ is $\mathrm{R}$-related to $y$, but $x$ is not $\mathrm{R}$-related to $x$ itself. Relations then add to properties in order to individuate. This option points clearly to a categorical approach to metaphysics, as we have suggested.

But perhaps one could argue that accepting a one-category approach to metaphysics is already going too far in a purely metaphysical direction; it could be claimed that by doing so we are not appreciating the real gist of the suggestion by Saunders that quantum particles are individuals. The suggestion, in this "less metaphysical approach", would be closer to the original Quinean setting in which Saunders' proposal originated. Due to that purely Quinean setting, involving regimentation in a first-order language and the methodological use of the PII as we commented in section 2.2, what we have is the traditional Quinean identification of being with entity (an object) and individuals. Once that is accepted, we are ready to apply Quine's criterion of ontological commitment (to be is to be the value of a variable) and read off the resulting metaphysics.

That really seems to be closer to what Saunders attempts at in (2003 and 2006). Given that, the issue is not one of providing a form of PII to ground a version of bundle theory according to a constituent ontology, but rather to apply the criterion of ontological commitment and to distinguish (in those papers, at least), fermions from bosons. Fermions are objects, bosons are not. In this sense, we have a clear instance of what Lowe $(2008,281)$ called a "no-category ontology". Obviously, it differs radically from the previous option (and from the options 
to come). It simply refuses to think of objects in terms of metaphysical categories. Everything is an object (because, there are just objects in the domain of quantifiers). That project seems to be clear from the following part of the conclusion in Muller and Saunders (2008, 541):

Fermions are discernible and are perfectly respectable as physical objects of predication and quantification in accordance with elementary logical syntax, even in a language in which identity is not primitive.

In other words, the real goal could be taken to show that weak discernibility implies that fermions are objects in this Quinean sense, and only that. Notice that following this option, however, even though we may be closer to the original proposal by Saunders, we get no closer to the original proposal by Muller (2011); that is, here we get no closer to OSR than M1 did. Here we have not the primacy of relations, in any ontological sense, a primacy that is typically advocated by defenders of OSR. More than that: relations do not feature in the ontology at all. In fact, that is the result of following the Quinean strictures of the proposal wherever they may lead. The relations are not among the values of the variables.

For the sake of reference later, let us call M2 this typical Quinean no-category ontology. It is also not structuralist, as Muller suggests it is imposed on us by quantum mechanics. However, it is also compatible with everything weak discernibility offers (it certainly is, given that it was the very first attempt at providing for weak discernibility in quantum mechanics; that is not all yet: the whole project of degrees of discernibility was first introduced by Quine, although his pioneer work was not related with quantum mechanics).

So, those positions M1 and M2 are not versions of OSR, although they are both compatible with weak discernibility and everything it brings to the metaphysics of entities in quantum mechanics. One is a robust version of bundle theory including relations in the scope of PII, the other is a no-category ontology. What else is required for us to have OSR?

\subsection{Structuralist ontologies}

Perhaps one of the first attempts to relate weak discernibility and OSR in quantum mechanics is to be found in Ladyman (2007). According to French (2011, 216), restricting the PII to a purely structuralist context as Ladyman does may prevent the application of the typical "skeptical tropes" against the attempt at grounding a thick form of individuality as in M1 (e.g. relations cannot ground individuality, relations cannot account for numerical difference, and so on). The features of this approach, however, shall provide for further packages in the metaphysical cart.

That one may take weak discernibility as providing for a version of ontic structural realism was stated as follows by Ladyman $(2007,31)$ :

The weak notion of individuality advocated by Saunders (according to which weak discernibility is sufficient for individuality) seems coherent. It would be question-begging to deny the sufficiency of weak discernibility merely because stronger forms of discernibility are sometimes available. Note however that while Saunders's view vindicates an ontology of individuals in the context of Quantum Mechanics, it is a thoroughly structuralist one in so far as objects are not assumed to be individuated independently of the nexus of relations in which they stand. Rather, they are contextually individuated. 
Ladyman believes $(2007,23)$ that weak discernibility is the best option for the naturalist philosopher willing to reject both non-individuality as well as some form of primitive intrinsic principle of individuality (i.e. again the TI principles). Those are precisely the horns comprising the metaphysical underdetermination, recall. According to Ladyman, TI principles seem to be rejected by physics, while he provides for no clear argument as to why we should not accept non-individuality. As for TI, the main reason for its rejection in quantum mechanics seems fairly simple: if the entities had TI, then one would be able to violate quantum permutation symmetry. Suppose we wish to describe a situation in which particles 1 and 2 are to be distributed between states A and B. In quantum mechanics, there is only one situation: one particle is in A and another is in B; permuting them makes no difference, there is no fact of the matter as to which particle is in which state. However, having TI would seem to preclude that possibility: because particles now have TI, even if we don't know which is the case, there is a situation in which we have particle 1 in $A$ and particle 2 in B, and a distinct situation in which we have particle 2 in A and particle 1 in B. That leads to the wrong kind of counting of the possibilities, with permutations giving rise to distinct states; thus, it seems, quantum mechanics violates TI (we shall not enter here in the merits of the argument).

But weak discernibility does more than providing for the best option on the metaphysics of quantum mechanics: adhering to weak discernibility bridges the gap between metaphysics and epistemology by levelling our metaphysics with our epistemology, so it is the natural choice for the naturalist $(2007,28)$. By the way, "bridging the gap between metaphysics and epistemology" is another motto by metaphysical naturalists; it may be put side by side with "tailoring the metaphysics to suit the physics" and Muller's "determination thesis".

However, mottos aside, how should we understand what is properly structural in this approach? The use of relations seems to be a good indication, but is not enough, as M1 and M2 indicate. How does a purely structural ontology differ from those non-structural approaches? A good way to address that issue is as follows: we assume that relations have ontological priority over objects, as friends of OSR have it, and try to spell that priority in some more specific terms. A most prominent way to do that is in terms of "ontological dependence", as first suggested in the context of OSR by French (2010).

Assuming the basic intuition that objects depend on the relations of a structure, French (2010, 104-106) advances three main kinds of dependence objects may have on structures:

- (Sym) Symmetric dependence: the identity of the putative objects is dependent on that of the relations of the structure and vice-versa.

- (Asy) Asymmetric dependence: the identity of the objects is asymmetrically dependent on the identity of the relations of the structure.

- (Ess) Essential dependence: the constitution (or essence) of the putative objects is dependent on the relations of the structure.

Now, each of those dependence relations is wholly compatible with distinct versions of OSR. Taking the relevant relations as involving at best weakly discerning relations does not help us to decide which kind of dependence relation to choose.

To begin with, (Sym) is the appropriate dependence relation to a form of Moderate Structural Realism (MSR), one conceding that both objects as well as structures exist on the same footing, with no strict priority of one over the other (see Esfeld and Lam 2008 and 2011). It is a structuralist metaphysics, however, according to Esfeld and Lam, because 
it is based on the claim that objects exist without intrinsic properties: nothing besides the relations characterize the objects; take the relations out and no feature remains, only what French $(2010,105)$ has called "bare relation bearers". The absence of intrinsic properties, thus, would be the structuralist ingredient resulting in "thin objects". So, overall, there are at least two ontological categories at work: particular objects and relations. Independently of how relations are conceived (universals or tropes), we have at least two categories in the context of a structuralist metaphysics.

Obviously, it is interesting to notice that Moderate Structural Realism is compatible with weak discernibility, but it could live very well with absolutely indiscernible entities as well. In fact, given that the particulars are on the same footing as the structures, then their numerical diversity does not depend on weak discernibility. What is relevant for us is that weak discernibility, for instance, does not rule out MSR, and is compatible with it. MSR could survive violations of the weak version of PII, but given that weak discernibility obtains in quantum mechanics, MSR also finds a place for it: it does weakly discern quantum entities. That is, as far as the symmetric dependence relation goes, nothing assures that the particulars will be discernible. When quantum mechanics is the theory providing for the structure, however, due to weak discernibility, those entities are seen as weakly discernible.

For the sake of further reference, let us call the previous view M3, comprising thus Moderate Structural Realism: objects and relations exist on the same footing, with objects and relations having a symmetric dependence relation.

Now, (Asy), on its turn, is the view attributed to Ladyman (2007) by French (2010). Recall that Ladyman explicitly uses weak discernibility to ground the identity of objects. Furthermore, Ladyman $(2007,28)$ believes that this approach is the correct option for those not adhering to an eliminativist version of structural realism, since it allows us to keep with individual objects. That is, one may keep objects in the metaphysics, but their identity is completely dependent on the identity of the relations.

The main idea by Ladyman, in this case, consists also in liberating objects from any kind of intrinsic identity characterizing thick approaches to individuality: objects need not be individuated neither by an intrinsic property nor by any intrinsic primitive principle (the TI principles we mentioned before). The identity of an entity does not remain unchanged outside the context of a structure: we simply cannot claim that one entity in a structure is the same as or different from another entity in another structure. Identity only makes sense inside a structure. In that sense, it is a strictly contextual individuality, grounding once again the existence of "thin objects".

Concerns may arise as to what are the objects whose identity is being contextually grounded. On the one hand, it seems that there is very little to prevent collapse of the view in either M1 or M3, depending on how the ideas of "thick" and "thin" objects are to be understood. On the other hand, there is also a metaphysical concern that what Ladyman is calling "thin individuals" may well play the role of the typical non-individuals (see Arenhart 2015 for the compatibility of weak discernibility and non-individuality). Typically, non-individuals may be understood as entities without identity conditions, where those identity conditions are cashed in terms either of intrinsic qualities or primitive intrinsic principles of individuality. It is in opposition to those principles that non-individuals are framed (see also French and Krause 2006, chap. 4 for the typical framing of non-individuality). So, this view could also be unpacked as reinforcing the non-individuals' horn of the underdetermination. 
Those questions attest to the fact that thin objects may be just too thin to be individuals in any metaphysically respectable sense. There is really little to be said about them, so the position may easily lend itself to other interpretations. Let us however concede, for the sake of argument, that those concerns may be dispelled in favor of a structuralist metaphysics comprising thin objects; the main problem, again, is that the notion of dependence, couched in terms of (Asy), as French $(2010,105)$ notes, also requires the postulation of bare property bearers. Notice, everything there is to the bare relata is obtained by the relations they enter into, even their very identity; then, of course, this identity depends of such relations, which determine a given context.

All those problems notwithstanding, for the sake of further reference, let us call this view M4. The main traits of M4 are: the existence of both relations and relata (the bare relata) and the idea of contextual individuality, granted by a notion of asymmetric dependence. In this sense, we have here also a two-category ontology: relations and (contextually individuated) bare relata, differing from M3 in the kind of dependence relation employed.

The third option on ontological dependence, employing essential dependence, is the choice for the eliminativist brand of OSR (French 2010 and 2011). According to the eliminativist, there are no objects, only the relations. The main motivation for this option is precisely the metaphysical underdetermination presented earlier: given that the nature of objects is underdetermined, we are better off eliminating objects completely. So, the appropriate dependence relation is (Ess).

The eliminativist adopts a one-category ontology: only the relations are primitive. Objects are completely derived from those relations (and monadic properties too). In opposition to the two structuralist views presented earlier, according to which objects exist somehow, here talk about objects is a mere pragmatic convenience, but reflects no ontological counterpart. That is, we can talk about objects without being ontologically committed with them.

Typically, eliminativists do not appeal directly to weak discernibility to motivate their position. On the contrary, non-eliminativists appeal to such relations in order to somehow break metaphysical underdetermination and keep objects, the same objects the eliminativist wishes to eliminate due to underdetermination. However, it is not forbidden by physics that the eliminativist, along with the appropriate dependence relation (Ess) could appeal to weak discernibility in order to ground her position. What is relevant for us is that weak discernibility does not prohibit that move, and that this is a further metaphysical package, let us call it M5, compatible with everything weak discernibility has to say about quantum mechanics.

Let us take stock and present the five metaphysical packages concerning the nature of the fundamental entities in quantum mechanics. All of them are available for the friend of weak discernibility, and as we shall argue, weakly discerning relations do not force any of them on us:

- (M1) One category ontology: there are relations, and objects are understood as constituted by bundles of relations, in a thick, substantial, sense. This is not a structuralist ontology, but rather a constitution ontology.

- (M2) No category ontology: objects are understood in a Quinean thin sense. There are just objects, and they are individuals just because being discernible somehow. This is also not a structuralist ontology. 
- (M3) Two category ontology: objects and relations are primitive. Objects are bare relation bearers, and there is a symmetric dependence relation between them. This is moderate ontic structural realism.

- (M4) Two category ontology: objects and relations are primitive. Objects are bare relation bearers, and there is an asymmetric dependence relation between them. Objects are contextually individuated.

- (M5) One category ontology: only relations exist. Objects are essentially dependent on relations. Eliminative ontic structural realism.

\section{Naturalism vindicated?}

To recall what was achieved in the previous section, let us state briefly our main findings there: as we have seen, there are at least five distinct metaphysical packages compatible with weak discernibility. So, there are no clear grounds on which the naturalist could hold that weak discernibility truly helps us determine the metaphysics. More than that, weak discernibility does not even unequivocally advance a structuralist metaphysics: at least two of the options are non-structural (M1 and M2), in the sense of not endorsing a metaphysics typically associated with the recently developed OSR. Three of them are structuralist metaphysics (M3, M4 and M5), or at least they were designed to be such, and should be understood as assuming relations as having ontological priority in some sense.

Our point in recalling those incompatible metaphysical readings was to bring to light that even in the context of weak discernibility there is much disagreement on the correct metaphysical understanding of the consequences of weakly discerning relations on what concerns quantum entities. So, the idea that weak discernibility helps us establish the PDT is incorrect, or, at least, there is still something missing if we are to establish that one of the contenders (or some further option not listed) is the correct one. But what could that further ingredient be? Obviously, the appeal to a purely metaphysical ingredient would not do to help the naturalist, so the further ingredient would have to show some naturalistically acceptable credentials, in a sense.

The first suggestion along these lines concerns the calling forth of some further aspect of science. That is, perhaps the underdetermination raised in the previous section may be broken with even more science. Here the idea of "bridging the gap between metaphysics and epistemology", as a tenet of metaphysical naturalism, plays a major role. There should be something else in science that could do the trick.

Muller seems to have something to offer along those lines, which he called scientific optimism $(2011,227)$, which states that:

Scientific optimism: science will provide us with everything that we can know about the physical world.

That seems a pretty clear suggestion that those problems should be answered in the context of the relevant science, viz. physics. Is there anything else physics could do to dispel at least some of the options available in the form of metaphysical packages M1-M5? Here perhaps we cannot expect any help in the same form as a derivation of weak discernibility: there is no direct sign or hint that could be derived from the quantum formalism that would enable us to break the underdetermination found in the last section. 
Anyway, the hope is that physics may help us. The problem is that it is not clear how that could be done. There is no simple appeal to physics that could clearly decide the issue. To see that, it is instructive to take a closer look at the kinds of arguments advanced by both defenders of eliminative versions of OSR (mainly Steven French), as well as by defenders of non-eliminative versions, having Moderate OSR and Ladyman's version on the other. Recall that non-eliminative versions of structural realism have to somehow posit bare relation bearers, while eliminativists will have no commitment with objects. Both sides argue for their positions, among other things, by taking their clues from physics.

Esfeld and Lam (2011, 148), among other purely logical and metaphysical objections against eliminativism, argue that eliminativism is just too revisionary, in the specific sense that it is revisionary beyond what is really required by current physics. There is no evidence coming from current physics requiring the elimination of objects, so, the argument goes, we should retain objects in our metaphysics. That move, as we mentioned, is intended not only to solve some of the logical and metaphysical problems raised by eliminativism (e.g. the claim that we cannot understand relations without relata, the claim that logic requires objects, and so on), but also to keep the metaphysics in step with physics.

Obviously, the claims that non-eliminativism has advantages on the metaphysical grounds over eliminativism should not concern the naturalist we are considering here: what is relevant is that the decision in favor of non-eliminativism is grounded on facts coming from our best science. So, we shall concern ourselves only with the argument that non-eliminativism is more coherent with current physics, which allegedly does not demand eliminativism (i.e. eliminativism goes beyond physics: it creates a gap between metaphysics and physics).

Now, if those kinds of appeal to physics were effective, and assuming that we could restrict ourselves to a structuralist metaphysics, we would have ruled out M5. That would not be enough; however, we would still have to decide between M3 and M4. Esfeld and Lam (2011) do that, choosing their own version of OSR over Ladyman's on metaphysical grounds (so, that can hardly count as an appeal to scientific optimism, as formulated previously). On the other hand, if we cannot grant that a structuralist metaphysics has preference over M1 and M2 (as it seems to be the case), then underdetermination is still even more worrying, and physics has even less control over the options.

So, even if we could grant that Esfeld and Lam's appeal to physics is effective, it does not help us effectively in breaking the underdetermination. And notice that this is a big "if". As we mentioned, there is no easy appeal to physics, not even to eliminate possible metaphysical packages such as M5. That is made clear because the eliminativist has an appeal to physics too, this time against non-eliminative versions of OSR! Let us see.

To present how the eliminativist may appeal to physics in order to motivate his view on objects, let us briefly recall that the non-eliminativist versions of OSR have to posit what French (2010) called "bare relation bearers". As the reader may already be guessing, the claim by French (2010, pp.105-106) is that for thin objects "to have any worth in this context, it needs a physical correlate and there is no physical correlate to this aspect of putative objects". That is, thin objects play no physical role, given that they depend on the relations and structures and are nothing by themselves. So, in the context of structural realism (adopting (Sym) or (Asy)), physics does not provide any content for those objects, so they are idle metaphysical posits, not required by physics.

According to French, then, the options for the friend of weak discernibility, in those cases, as we have already mentioned, is either to adhere to a robust notion of object as in 
M1, or else to forget about objects and accept M5. We could add to that M2, an option not considered by French. As French sees the issue, M1 would feed underdetermination, while M5 is motivated traditionally not to break underdetermination, but to overcome it. Either way, the answer was not taken from physics, and underdetermination was not broken. Also, from the point of view of the naturalist adopting scientific optimism, the situation is still left with a whole bunch of metaphysical options available, and no clearly physical evidence in favor of one of them. So, metaphysical naturalism wedded to scientific optimism seems to receive little comfort from physics in this case.

It seems clear that our attempt at reading the scientific optimism condition rather strictly led us to a debate that resembles very closely metaphysical debates. The dispute was led by both sides claiming that physics does not support the opponents' views (there is a gap between metaphysics and physics). Perhaps our strict reading of the claim in favor of scientific optimism would lead us not to PDT, but rather to some form of quietism on what concerns metaphysical issues (see also Wolff 2015 for further discussion of the connection between naturalism and realism that may be useful in this context). That is, noticing that the discussion cannot be decided by science and sticking to the idea that knowledge of physical reality has this only source, the naturalist could, in this case, avoid involving herself with such metaphysical disputes until further evidence is presented. That, however, is far from the conclusion we were expecting when we suggested scientific optimism could help us break underdetermination.

But what if scientific optimism should be taken more lightly, meaning that we should rely on scientific investigation taken in a broader sense? That is: perhaps the underdetermination could be broken by appeal to non-empirical virtues typically involved in theory choice. That would still be a way of importing scientific methods to metaphysical disputes, right?

That seems correct, but the move takes us far from the core claim of scientific optimism. If we allow the possibility that the dispute could be decided by non-empirical virtues such as simplicity, economy and, perhaps, maintenance of the greatest number of tenets of common sense as possible (to mention just a few), then we are not far from claiming that naturalists should decide their issues appealing to virtues that are also typical in disputes in metaphysics, which are also judged according to those virtues. As Morganti (2014) has put it, the metaphysician is already used to such disputes, given that the choice of theory in metaphysics is performed on other grounds than merely empirical adequacy (although empirical adequacy is of great relevance for a metaphysical theory too, of course).

So, that kind of move would have as a consequence that the debate in most contemporary metaphysics is also conducted on naturalistically acceptable grounds. That reduces the advantage of the naturalist over traditional metaphysicians to no advantage at all. More than that, given that both in science and in metaphysics the dispute over the precise role of those virtues is largely unsettled, we seem to have no reason to think that naturalism, if it really means just that, have added anything else to the current debate. So, either it is no news, or else it leads to a form of quietism. Both options are no comfort for someone expecting to solve metaphysical underdetermination.

\section{Final remarks}

As we have seen, the idea that science may help us decide puzzling metaphysical issues concerning the nature of quantum entities is rather tempting. Weak discernibility seems to 
provide hope to this idea, while supporting the claim that a version of the PII is correct in quantum mechanics. Against the tradition, which saw the PII as failing, weak discernibility shows that a version of PII holds in the theory. The natural question is: can we advance a further step, so that weak discernibility could be seen as providing help for us to determine the metaphysics?

The thesis of the determination of the metaphysics by physics was advanced precisely to show how such a help from weak discernibility may be achieved. The result, as Muller (2011) claimed, was a structural metaphysics. So is it the end of underdetermination? Not really. As we have seen, there are not only at least three distinct versions of OSR which may with equal rights claim to have been derived from weak discernibility, but also two other options that are clearly non-structural, at least one of them coming from Saunders, the pioneer of weak discernibility in quantum mechanics. So, underdetermination plagues weak discernibility.

There are no other easy approaches to breaking underdetermination coming from science. As we have seen, both eliminativists as well as non-eliminativists versions of OSR could hold that physics supports their view, while attacking the opponent for not being compatible with physics. What is left for us is the old style of debate, contrasting virtues such as simplicity and ontological economy, a constant trade looking for a balance between the required posits and their explanatory power. This, however, is what typical metaphysical debates already do.

\section{REFERENCES}

Ainsworth, Peter. M. 2010. What is ontic structural realism? Studies in History and Philosophy of Modern Physics 41: 50-57.

Arenhart, Jonas R. Becker. 2012. Ontological frameworks for scientific theories. Foundations of Science 17: 339-356.

-. 2015. The received view on quantum non-individuality: Formal and metaphysical analysis. Synthese, DOI 10.1007/s11229-015-0997-5.

Chakravartty, Anjan. 2013. On the prospects of naturalized metaphysics. In Scientific Metaphysics, eds. Don Ross, James Ladyman and Harold Kincaid, 27-50. Oxford: Oxford University Press.

Esfeld, Michael, and Lam, Vincent. 2008. Moderate structural realism about space-time. Synthese 160: 27-46.

-. 2011. Ontic Structural Realism as a Metaphysics of Objects. In Scientific Structuralism, eds. Peter Bokulich and Alisa Bokulich, 143-159. Dordrecht: Springer.

French, Steven. 2010. The interdependence of structure, objects, and dependence. Synthese 175: 177-197.

-. 2011. Metaphysical underdetermination: Why worry? Synthese, 180: 205-221.

French, Steven, and Krause, Décio. 2006. Identity in Physics. A historical, philosophical and formal analysis. Oxford: Oxford University Press.

Friebe, Cord. 2014. Individuality, distinguishability, and (non-)entanglement: A defense of Leibniz's Principle. Studies in History and Philosophy of Modern Physics 48: 89-98.

Huggett, Nick, and Norton, Joshua. 2014. Weak discernibility for quanta, the right way. British Journal for the Philosophy of Science 65: 39-68.

Krause, Décio, and Arenhart, Jonas R. Becker. 2015. Individuality, quantum physics, and a metaphysics of nonindividuals: The role of the formal. In Individuals Across the Sciences, eds. Alexandre Guay and Thomas Pradeau, 61-80. Oxford: Oxford University Press. 
Ladyman, James. 2007. On the identity and diversity of objects in a structure. Proceedings of the Aristotelian Society Supplementary Volume LXXX: 23-43.

Ladyman, James, and Bigaj, Thomasz. 2010. The Principle of the Identity of Indiscernibles and Quantum Mechanics. Philosophy of Science 77: 117-136.

Ladyman, James, Linnebo, Øystein, and Pettigrew, Richard. 2012. Identity and discernibility in philosophy and logic. Review of symbolic logic 5(1): 162-186.

Lowe, E. Jonathan. 2008. New directions in metaphysics and ontology. Axiomathes 18: 273-288.

Maudlin, Tim. 2007. The Metaphysics Within Physics. Oxford: Oxford University Press.

Morganti, Matteo. 2014. Objects and structures: Some (further) remarks concerning the motivation for ontic structuralism and its metaphysical characterization. Methode 4: 77-97.

Muller, Fred A. 2011. Withering away, weakly. Synthese, 180(2): 223-233.

-. 2014. Elementary particles and metaphysics. In New directions in the philosophy of science. The philosophy of science in a European perspective 5, eds. Maria Carla Galavotti et. al., 417-431. Dordrecht: Springer.

Muller, Fred A., and Saunders, Simon. 2008. Discerning Fermions. British Journal for the Philosophy of Science 59: $499-548$.

Muller, Fred A. and Seevinck, Michael P. 2009. Discerning Elementary Particles. Philosophy of Science 76(2): 179-200.

Saunders, Simon. 2003. Physics and Leibniz's Principles. In Symmetries in Physics: Philosophical Reflections, eds. Katherine Brading and Elena Castellani, 289-307. Cambridge: Cambridge University Press.

-. 2006. Are Quantum Particles Objects? Analysis 66: 52-63.

Wolff, Johanna. 2015. Naturalistic quietism or scientific realism? Synthese, DOI 10.1007/s11229-0150873-3.

Jonas R. Becker Arenhart earned a PhD in Epistemology and Logic at the Federal University of Santa Catarina, Florianópolis, Brazil. He is currently associate professor in the Philosophy Department at the Federal University of Santa Catarina. His main areas of research are philosophy of logic, philosophy of science, and the metaphysics of science, focusing mainly in the metaphysics of quantum mechanics. Among papers in international philosophy journals, most recent publications include the book The Logical Foundations of Scientific Theories, published in 2016 by Routledge, co-authored with Décio Krause.

Address: Federal University of Santa Catarina. Department of Philosophy. Florianópolis, Santa CatarinaBrazil. E-mail: jonas.becker2@gmail.com 